\title{
Evaluation of Life Quality in Patients with Oral Mucosal Lesions
}

\author{
${ }^{1}$ Maryam Baharvand, ${ }^{2}$ Hossein Karami, ${ }^{3}$ Hamed Mortazavi
}

\section{ABSTRACT}

Aim: The mouth does many functions, such as speaking, chewing, taste perception and swallowing. Therefore, any pathologic changes of oral mucosa might affect patients' life quality. The aim of this study was to investigate quality of life (QoL) in patients with oral mucosal lesions.

Materials and methods: A total of 40 patients with oral mucosal lesions attending at Oral Medicine Department of Shahid Beheshti University of Medical Sciences Dental School entered in a cross-sectional study. Variables, such as age, sex, level of education, kind of lesions, systemic disease and medicine consumptions were recorded in data sheets, and QoL was assessed using European Organization for Research and Treatment of Cancer Quality of Life Questionnaire - Head and Neck 35 (EORTC-QLQ-H\&N35). Data were analyzed by Statistical Package for the Social Sciences (SPSS) software (version 16), backward regression model, student's t-test and Chi-square test.

Results: Out of 40 patients with mean age of (44 \pm 13$)$, $25(62.5 \%)$ were women and $15(37.5 \%)$ were men. The mean score of life quality was $43.8 \pm 9.8$ (range of score: $30-120$ ), whereas in mostly frequent oral lesions it was reported to be $41.7 \pm 6.4$ (lichen planus), $40.8 \pm 6.7$ (mucocele), $42.4 \pm 7.2$ (irritation fibrosis), respectively. Regression analysis showed that higher levels of education affect quality of life and its aspects of pain, sexual function, xerostomia, swallowing and speaking negatively. Recurrent aphthous stomatitis deteriorated aspects of pain, and eating of QoL. However, irritation fibrosis and mucocele aggravated QoL in terms of swallowing and social relationship respectively.

Conclusion: Oral mucosal lesions deteriorate QoL mildly. Different dimensions of life quality can be affected by recurrent aphthous stomatitis, irritation fibrosis and mucocele.

Keywords: Quality of life, Mouth diseases, Oral mucosa.

How to cite this article: Baharvand $\mathrm{M}$, Karami $\mathrm{H}$, Mortazavi $\mathrm{H}$. Evaluation of Life Quality in Patients with Oral Mucosal Lesions. Int J Experiment Dent Sci 2014;3(1):29-32.

Source of support: Nil

Conflict of interest: None declared

\footnotetext{
${ }^{1,3}$ Associate Professor, ${ }^{2}$ Private Practitioner

${ }^{1,3}$ Department of Oral Medicine, Dental School, Shahid Beheshti University of Medical Sciences, Tehran, Iran

${ }^{2}$ Private Practice, Iran
}

Corresponding Author: Hamed Mortazavi, Associate Professor, Department of Oral Medicine, Dental School, Shahid Beheshti University of Medical Sciences, Tehran, Iran, Phone: 00982129902311, e-mail: hamedmortazavi2013@gmail.com

\section{INTRODUCTION}

Oral mucosal lesions have been reported with a wide range of prevalence rate among different populations. ${ }^{1-3}$ Early diagnosis and treatment of oral lesions can decrease the resultant complications such as psychological and economic impacts on patients, their families and the community. ${ }^{4,5}$ The term quality of life $(\mathrm{QoL})$ was first described by Arthur Cecil Pigou in 1920. The term is used in a wide range of contests including the field of healthcare. ${ }^{6}$ World Health Organization (WHO) generally defines QoL as people's perception of their life situation according to their culture and appraisal criteria. More recent descriptions about life quality focus on the difference between one's expectation and the reality, the more the discrepancy the less the QoL. ${ }^{7,8}$

Method to measure life quality differs in terms of different field of science, e.g. psychologists use 'life satisfaction scale', and health practitioners apply some questionnaires like 'health related QoL'. .910 To date, there are a few researchers addressing QoL in oral health problems. ${ }^{7,10-12}$

Locker evaluated the relationship between oral disorders and change in QoL, and showed that overall 19.5\% of individuals reported one or more impacts 'fairly often' or 'very often'. Of these, $48.3 \%$ reported being bothered by these impacts, $40.3 \%$ mentioned that their life was totally affected, and $36.0 \%$ cited that their QoL was affected. ${ }^{13}$ None of the previous studies addressed how much each entity affects various aspects of life quality. This study was designed to assess the impact of oral mucosal lesions on QoL totally and its diverse dimensions in Shahid Beheshti Dental School.

\section{MATERIALS AND METHODS}

In a cross-sectional study, 40 consecutive dental patients with ulcerative or exophytic oral lesions referred to Oral Medicine Department were studied. Every patient who was 18-year-old or above, and having literacy to read and write Farsi language entered the study. Patients with debilitating disease affecting life quality, those on drug therapy with known adverse effects on oral mucosa, such as dry mouth, dysgeusia, and mucosal desquamation, and patients having bony lesions were excluded from the study. Every patient undergone complete oral examination and having final diagnosis of his/her lesion, was asked to fill out the questionnaire of European Organization for Research and 
Treatment of Cancer Quality of life Questionnaire-Head and Neck 35 (EORTC-QLQ-H\&N35) to assess the QoL related to oral condition and function. The above mentioned questionnaire contained 35 queries about different aspects of QoL. Each question had four answers: 'absolutely not', 'a few', 'relatively high' and 'completely high' with the attributed scores of 1, 2, 3 and 4, respectively. The first 16 questions were related to oral signs and symptoms, and the next 14 were pertinent to interpersonal and social relationships. The last 5 questions with yes-no answers were related to the complications of cancer and cancer therapy; therefore they excluded from the statistical analysis. The Kronbach's coefficient was measured for the remaining 30 questions to be 0.89 . Totally, seven aspects of life quality were evaluated by EORTC-QLQ-H\&N35 in our patients: pain (q 1-4), swallowing (q 5-8), xerostomia (q 11, 12), eating (q 19, 20), speaking (q 22, 23), socializing (q 24-27), and sexual life (q 28, 29). The minimum and maximum scores of the questionnaire were 30 and 120 respectively, which the higher scores were indicative of lower QoL. The patients' demographics, characteristics of lesions and their QoL scores were recorded in data forms. Statistical analysis was accom-

Table 1: Mean score of QoL in different oral mucosal lesions

\begin{tabular}{llll}
\hline Lesion & Frequency & Percent & Mean \pm SD \\
\hline Lichen planus & 10 & 25 & $41.7 \pm 6.4$ \\
Mucocele & 6 & 15 & $40.8 \pm 6.7$ \\
Irritation fibrosis & 6 & 15 & $42.4 \pm 7.2$ \\
Peripheral giant cell & 3 & 7.5 & $40.2 \pm 4.8$ \\
granuloma & & & \\
Leukoplakia & 3 & 7.5 & $36.2 \pm 7$ \\
Recurrent aphthous & 4 & 10 & $50.2 \pm 8.5$ \\
stomatitis & & & \\
Fissured tongue & 3 & 7.5 & $38.2 \pm 6.4$ \\
Epulis granulomatosum & 1 & 5 & 41 \\
Epulis fissuratum & 1 & 2.5 & 40 \\
Adenoid cystic carcinoma & 1 & 2.5 & 42 \\
Squamous cell carcinoma & 1 & 2.5 & 43 \\
Pemphigus vulgaris & 1 & 2.5 & 38 \\
\hline Total & 40 & 100 & \\
\hline
\end{tabular}

SD: Standard deviation plished using Chi-square test, Student's t-test and Backward regression by means of SPSS software version 16. p-value less than 0.05 was considered to be significant.

\section{RESULTS}

A total of 40 patients including 25 women $(62.5 \%)$ and 15 men (37.5\%) with the mean age of $44 \pm 13$ were examined and interviewed. Out of them, $10(25 \%)$ had literacy to read and write, $12(30 \%)$ were high-school graduates, $12(30 \%)$ had BS degree, and the remainder $6(15 \%)$ had postgraduate degrees. The mean score of QoL among the patients was $43.8 \pm$ 9.8. Table 1 demonstrates QoL scores in different oral mucosal lesions. The highest scores (the worst) of QoL were obtained by patients with recurrent aphthous stomatitis (RAS) and irritation fibrosis, and the least (the best) were reported by leukoplakia patients. The mean scores of life quality and its different aspects in terms of sex and level of education were shown in Table 2. In this regard, men compared to women and highly-educated patients in comparison to people with moderate to low levels of education obtained higher scores (lower QoL).

Using regression model and backward analysis, the role of variables, such as age, sex, level of education, and type of oral lesions on total QoL score and its different aspects were analyzed. For this purpose, oral lesions were compared to RAS, (due to its more negative effects on QoL) so that if the coefficient of a disease (B) tend to be negative and $p<$ 0.05 , the scale of the disease will be lower than RAS, and the relating QoL be better. It is noteworthy, that literacy to read and write and high-school graduation were compared to academic education. As shown in Table 3, the only variable with significant effect on total score of QoL was level of education $(p=0.002)$. According to this table, the following results regarding the effects of different variables on seven aspects of QoL were yielded:

1. There was a significant relationship between pain aspect in mucocele $(\mathrm{p}=0.004, \mathrm{~B}=-3.825)$ and lichen planus $(\mathrm{p}=0.032, \mathrm{~B}=-1.406)$, and ability to read and write

Table 2: Scores of QoL aspects (mean \pm SD) in different sexes and levels of education

\begin{tabular}{llllllll}
\hline QoL aspects & Male & Female & Total & $\begin{array}{l}\text { Read/write } \\
\text { ability }\end{array}$ & $\begin{array}{l}\text { High school } \\
\text { graduation }\end{array}$ & $\begin{array}{l}\text { Academic } \\
\text { degree }\end{array}$ & Total \\
\hline Pain & $7.7 \pm 1.9$ & $6.6 \pm 2.2$ & $6.7 \pm 2.1$ & $5.4 \pm 1.2$ & $7.2 \pm 2.3$ & $7.1 \pm 2.1$ & $6.7 \pm 2.1$ \\
Swallowing & $6.2 \pm 1.4$ & $5.6 \pm 1.7$ & $5.8 \pm 1.6$ & $5 \pm 1$ & $6.1 \pm 1.8$ & $6.1 \pm 1.6$ & $5.8 \pm 1.6$ \\
Xerostomia & $1.6 \pm 0.8$ & $1.2 \pm 0.4$ & $1.4 \pm 0.6$ & $1.2 \pm 0.4$ & $1.4 \pm 0.5$ & $1.5 \pm 0.8$ & $1.4 \pm 0.6$ \\
Eating & $8 \pm 2.5$ & $7.8 \pm 3$ & $7.9 \pm 2.9$ & $7.2 \pm 2.6$ & $8 \pm 2.8$ & $8.3 \pm 3.2$ & $7.9 \pm 2.9$ \\
Speaking & $2.8 \pm 1.5$ & $2.3 \pm 0.7$ & $2.5 \pm 1.1$ & $2.3 \pm 0.6$ & 2 & $3 \pm 1.4$ & $2.5 \pm 1.1$ \\
Social life & $5.6 \pm 2.7$ & $4.4 \pm 1$ & $4.9 \pm 1.9$ & 4 & $4.5 \pm 1$ & $5.7 \pm 2.5$ & $4.9 \pm 1.9$ \\
Sexual life & $2.6 \pm 0.9$ & $2.5 \pm 1$ & $2.5 \pm 1$ & $2.2 \pm 0.6$ & $2.5 \pm 1.2$ & $2.8 \pm 0.9$ & $2.5 \pm 1$ \\
\hline Total QoL & $46.5 \pm 12.5$ & $42.3 \pm 7.7$ & $43.8 \pm 9.8$ & $43.4 \pm 5^{* *}$ & $34.6 \pm 9.6^{* * *}$ & $46.9 \pm 10$ & $43.8 \pm 9.8$ \\
scores & & & & & & & \\
\hline
\end{tabular}

${ }^{*} p=0.494,{ }^{* *} p=0.002,{ }^{* * *} p=0.208$, SD: Standard deviation 


\begin{tabular}{|c|c|c|c|c|c|c|c|c|c|c|c|c|c|c|c|c|}
\hline \multirow{2}{*}{$\begin{array}{c}\text { Variable } \\
\text { QoL aspect }\end{array}$} & \multicolumn{2}{|c|}{ Sex } & \multicolumn{2}{|c|}{ Age } & \multicolumn{2}{|c|}{$\begin{array}{c}\text { Read/write } \\
\text { ability }\end{array}$} & \multicolumn{2}{|c|}{$\begin{array}{l}\text { High school } \\
\text { graduation }\end{array}$} & \multicolumn{2}{|c|}{ Irritation fibrosis } & \multicolumn{2}{|c|}{ Lichen planus } & \multicolumn{2}{|c|}{ mucocele } & \multicolumn{2}{|c|}{ Other lesions } \\
\hline & $\beta$ & Sig & $\beta$ & Sig & $\beta$ & Sig & $\beta$ & Sig & $\beta$ & Sig & $\beta$ & Sig & $\beta$ & Sig & $\beta$ & Sig \\
\hline Pain & 0.87 & 0.265 & -0.044 & 0.305 & -2.440 & 0.001 & $0 . .090$ & 0.927 & 0.604 & 0.741 & -1.406 & 0.032 & -3.825 & 0.004 & 0.764 & 0.743 \\
\hline Sexual life & -0.281 & 0.421 & 0.022 & 0.319 & -1.691 & 0.012 & -6.074 & 0.129 & 0.834 & 0.263 & 0.825 & 0.252 & 7.205 & 0.332 & 0.074 & 1.140 \\
\hline Speaking & 0.302 & 0.794 & -0.008 & -0.749 & -0.953 & 0.027 & -1.248 & 0.041 & -0.515 & 0.569 & 0.010 & 0.991 & -0.691 & 0.412 & 0.279 & 1.033 \\
\hline Eating & 0.764 & 0.755 & 0.038 & 0.592 & -1.307 & 0.533 & 0.459 & 0.737 & -2.970 & 0.230 & -5.735 & 0.030 & -5.894 & 0.008 & 0.171 & -3.559 \\
\hline Xerostomia & -0.032 & 0.941 & 0.018 & 0.505 & -1.703 & 0.189 & -1.735 & 0.028 & 0.747 & 0.396 & 0.501 & 0.569 & -0.733 & 0.604 & 0.664 & 0.695 \\
\hline Swallowing & 0.289 & 0.649 & 0.028 & 0.994 & -1.059 & 0.048 & -0.808 & 0.920 & 1.612 & 0.019 & -0.331 & 0.799 & -1.432 & 0.187 & 0.928 & 0.127 \\
\hline
\end{tabular}

$(\mathrm{p}=0.001, \mathrm{~B}=-2.440)$. This means that lichen planus patients (compared to RAS) and those with lower education level (compared to academic education) had better QoL in terms of pain.

2. A significant relationship was found between QoL in sexual life aspect and ability to read and write $(p=0.012$, $\mathrm{B}=-1.691)$, i.e. lower educated people had better life quality compared to academic graduates in terms of sexual life.

3. It was noticed that a significant relationship exists between mucocele $(p=0.036, B=-1.877)$ and social dimension of life quality. Therefore, those involved by mucocele experienced worse social life compared to RAS patients.

4. A significant relationship was noted between speaking aspect of life quality with high-school graduation $(\mathrm{p}=0.041, \mathrm{~B}=-1.248)$ and ability to read and write $(\mathrm{p}=0.027, \mathrm{~B}=-0.953)$ both compared to academic education. This means that lower educated people had fewer difficulties in speaking aspect when involved with oral mucosal lesions than well educated ones.

5. There was a significant relationship between QoL in terms of eating with lichen planus $(p=0.030, B=-5.735)$ and mucocele $(\mathrm{p}=0.008, \mathrm{~B}=-5.894)$ both compared to RAS. As a result, lichen planus and mucocele deteriorated eating aspect less than RAS.

6. A significant relationship was found between xerostomia aspects of QoL and graduation from high school ( $\mathrm{p}=$ $0.028, \mathrm{~B}=-1.735$ ) compared to academic education. Then high school graduates were less affected by xerostomia than academic patients.

7. A significant relationship was noted between swallowing aspect of life quality and irritation fibrosis $(p=0.019$, $\mathrm{B}=-1.612$ ), i.e. irritation fibrosis caused more negative effect on swallowing aspect compared to RAS.

\section{DISCUSSION}

In this study, QLQ was evaluated using Farsi version of EORTC-QLQ-H\&N35, a standard questionnaire, which has been already used in previous studies. ${ }^{10,14,15}$

All the patients in current study were above 20 years to assure they have enough perception about QoL questions like John's study. ${ }^{11}$ The mean score of life quality among our patients was $43 \pm 98$ which was in accordance with Locker's. ${ }^{13}$ With regard to the range of scores which was between 30 and 120, it seems that oral mucosal lesions apply minor impact on QoL. Due to lack of a cut-off point for QoL scale, the comparison was based on the minimum and maximum of scores.

Recurrent aphthous stomatitis worsened life quality more than other oral lesions may be due to its pain and discomfort imposed on patients.

Men had lower QoL than women when affected by oral lesions. This was true when considering all of different aspects of life quality, i.e. pain, sexual life, social life, eating, swallowing, speaking and xerostomia. Since men usually seek medical and dental care later, they are more likely to be affected by disease complications and thus experience lower QoL. Similar results were yielded in Klage's and John's studies. ${ }^{11,12}$

It was noticed that highly educated patients had lower QoL in comparison to people with moderate or lower levels of education.

We used regression analysis to assess the effects of different variables in the study on seven dimensions of life quality. Regarding 'pain aspect', patients with RAS had worse life quality than those with mucocele and lichen planus, may be due to the painful nature of RAS. Patients with lichen planus and mucocele had better QoL in 'eating' aspect compared to RAS patients, i.e. RAS patients had more difficulties in eating. Highly educated patients with oral lesions had more deteriorated life quality in terms of 'sexual function', 'xerostomia', 'swallowing', and 'speaking' similar to Gerritse's study. ${ }^{16}$ Patients having mucocele reported worse QoL in 'social aspect'. Mucocele is most frequently seen in the lower lip and can cause esthetic problems and make patients avoid socializing. ${ }^{17}$ On the other hand, irritation fibrosis disturbed 'swallowing' aspect of life quality more than RAS.

For more conclusive results, it is suggested that similar studies be accomplished in greater samples of population. Meanwhile, it is recommended that QoL be surveyed before and after treatment of oral lesions. 
In conclusion, oral mucosal lesions affect QoL mildly. RAS, irritation fibrosis and mucocele deteriorated aspects of pain, swallowing, and socializing of life quality, respectively.

\section{ACKNOWLEDGMENTS}

This article was based on an undergraduate thesis \# 3072 which was successfully accomplished by Hossein Karami under the supervision of Dr Maryam Baharvand in academic year of 2011 to 2012 in Shahid Beheshti University of Medical Sciences Dental school.

The authors are greatly thankful to the patients and the staff members of the Department of Oral and Maxillofacial Medicine for their inmost cooperation.

\section{REFERENCES}

1. Demko CA, Sawyer D, Slivka M, Smith D, Wotman S. Prevalence of oral lesions in the dental office. Gen Dent 2009;57(5):504-509.

2. Castellanos JL, Díaz-Guzmán L. Lesions of the oral mucosa: an epidemiological study of 23785 Mexican patients. Oral Surg Oral Med Oral Pathol Oral Radiol Endod 2008;105(1):79-85.

3. Jahanbani J, Sandvik L, Lyberg T, Ahlfors E. Evaluation of oral mucosal lesions in 598 referred Iranian patients. Open Dent J 2009;3:42-47.

4. Espinoza I, Rojas R, Aranda W, Gamonal J. Prevalence of oral mucosal lesions in elderly people in Santiago, Chile. J Oral Pathol Med 2003;32(10):571-575.

5. Martínez Díaz-Canel AI, García-Pola Vallejo MJ. Epidemiological study of oral mucosa pathology in patients of the Oviedo School of Stomatology. Med Oral 2002;7(1):4-9.

6. Baharvand M, Shoalehsaadi N, Barakian R, Moghaddam EJ. Taste alteration and impact on quality of life after head and neck radiotherapy. J Oral Pathol Med 2013;42(1):106-112.
7. World Health Organization. WHO Disability Assessment Schedule II (WHODAS II) WHOQOL Study Protocol. WHO (MNH7PSF/93.9). 1993. Available at: http://www.who.int/icidh/ whodas/index.html (accessed 18 December 2009).

8. Menon B, Cherkil S, Aswathy S, Unnikrishnan AG, Rajani G. The process and challenges in the translation of world health organization quality of life (WHOQoL- BREF) to a Regional Language; Malayalam. Ind J Psychol Med 2012;34(2):149-152.

9. Velikova G, Brown JM, Smith AB, Selby PJ. Computer-based quality of life questionnaires may contribute to doctor-patient interactions in oncology. Br J Cancer 2002;86(1):51-59.

10. Zotti P, Lugli D, Vaccher E, Vidotto G, Franchin G, Barzan L. The EORTC quality of life questionnaire-head and neck 35 in Italian laryngectomized patients. European organization for research and treatment of cancer. Qual Life Res 2000;9(10):1147-1153.

11. John MT, Koepsell TD, Hujoel P, Miglioretti DL, LeResche L, Micheelis W. Demographic factors, denture status and oral health-related quality of life. Community Dent Oral Epidemiol 2004;32(2):125-132.

12. Klages U, Bruckner A, Zentner A. Dental aesthetics, self-awareness, and oral health-related quality of life in young adults. Eur J Orthod 2004;26(5):507-514.

13. Locker D, Quiñonez C. To what extent do oral disorders compromise the quality of life? Community Dent Oral Epidemiol 2011;39(1):3-11.

14. Osthus AA, Aarstad AK, Olofsson J, Aarstad HJ. Head and neck specific health related quality of life scores predict subsequent survival in successfully treated head and neck cancer patients: a prospective cohort study. Oral Oncol 2011;47(10):974-979.

15. Baharvand M, Sarrafi M, Alavi K, Jalali Moghaddam E. Efficacy of topical phenytoin on chemotherapy-induced oral mucositis; a pilot study. Daru 2010;18(1):46-50.

16. Gerritsen AE, Allen PF, Witter DJ, Bronkhorst EM, Creugers NH. Tooth loss and oral health-related quality of life: a systematic review and meta-analysis. Health Qual Life Outcomes 2010;8:126.

17. Bahadure RN, Fulzele P, Thosar N, Badole G, Baliga S. Conventional surgical treatment of oral mucocele: a series of 23 cases. Eur J Paediatr Dent 2012;13(2):143-146. 